\title{
»Ein Hauch von Mystizismus" Hofmannsthals Scheitern an Goldoni in der Komödie "Cristinas Heimreise«
}

\author{
Goldoni, le deux Gozzi et la Rosalba, \\ Guardi et le Buranello, Da Ponte, Casanova, \\ les gazettes, les Granelleschi, tout cela, tous ceux-ci, \\ en minatures et mélodies, en comédies et chansonnettes, \\ en tableautins, en escapades et lestes choses \\ s'accordent à proclamer une nuance d'âme qui fut heureuse. \\ Philippe Monnier, Venise au XVIII ${ }^{e}$ siècle (1907)
}

\section{Einleitung}

Dass Venedig in Hofmannsthals Komödien nicht nur eine beliebte $\mathrm{Ku}-$ lisse, sondern auch einen zentralen sowie ausdrücklich ’literarischen Themenkomplex darstellt, davon zeugt nicht zuletzt der Umstand, dass Hofmannsthal bestimmende Anregungen zur Komödiendichtung um 1907 gerade vom Venezianer Carlo Goldoni bekommt; Goldonis Komödien hinterlassen auch später im Werk Hofmannsthals deutliche Spuren. ${ }^{1}$ Tatsächlich ist die neuzeitliche europäische Komödientradition, die auf die Commedia dell'Arte zurückgeht und sich dann im 18. Jahrhundert in Goldonis Theaterreform weiterentwickelt, für Hofmannsthal neben

1 Goldoni-Bezüge, die in der Kritischen Ausgabe nachgewiesen sind, finden sich außer in "Cristinas Heimreise" etwa auch im 1907 begonnenen Komödienfragment "Silvia im »Stern« (SW XX Dramen 18), im ebenfalls seit 1907 konzipierten "Andreas«-Roman (SW XXX Roman) sowie in der 1909/10 konzipierten Operndichtung "Der Rosenkavalier " (SW XXII Dramen 20). Hinweise auf Goldoni ziehen sich durch Hofmannsthals gesamtes Schaffen, wie seine Aufzeichnungen belegen; Einträge zu Goldoni findet man bis in die 1920er Jahre (vgl. etwa SW XXXVIII Aufzeichnungen, Text, S. 920). Bereits 1892 erwähnt er "La locandiera« (ebd., S. 158 und S. 163); an Marie von Gomperz schreibt er am 13./14. Mai 1892: "Die Duse fällt gerade in meine Prüfungswoche; in Cleopatra und Locandiera, einem wirklich italienischen Stück von Goldoni, werde ich sie mir aber jedenfalls ansehen." (BW Gomperz, S. 69f.) Auch sonst wird Goldoni in Briefen zwischen 1892 und 1924 immer wieder erwähnt. 
dem Wiener Volkstheater prägend gewesen. ${ }^{2}$ In den 1980er Jahren ist Roger Bauer zum ersten Mal den Bezügen von Hofmannsthals Komödien zur venezianischen Tradition nachgegangen und hat sich dabei nicht zuletzt mit Goldoni beschäftigt. ${ }^{3}$ Doch seitdem hat die Forschung den Themenkomplex Hofmannsthal und Goldoni nur wenig beachtet. ${ }^{4}$ An Bauers Studien anknüpfend, nimmt sich dieser Aufsatz vor, das wichtige Thema weiterzuverfolgen, wobei ein besonderes Augenmerk auf »Cristinas Heimreise « liegen soll.

Nach einem ersten Abschnitt, in dem Hofmannsthals Goldoni-Rezeption umrissen wird, konzentriert sich der Beitrag in einem zweiten Teil auf die Bezüge zwischen "Cristinas Heimreise" und Goldonis "La locandiera« (dt. »Mirandolina«), uraufgeführt 1752 im Teatro Sant'Angelo in Venedig. Dabei werden besonders die erste und die zweite Fassung von Hofmannsthals Komödie untersucht. Diese Komödie Goldonis hatte Hofmannsthal 1892 - genau in dem Jahr, in dem er auch zum ersten Mal nach Venedig fuhr - in der Aufführung mit der bekannten Schauspielerin Eleonora Duse im Wiener Burgtheater gesehen. "La locandiera« gehört außerdem zu den Texten Goldonis, die Hofmannsthal besaß und die sich, mit Annotationen versehen, welche sich explizit auf »Cristinas Heimreise« beziehen, noch heute unter den überlieferten Beständen seiner Bibliothek befinden. ${ }^{5}$ In einem abschließenden Fazit wird der

2 Vgl. dazu auch Hans-Albrecht Koch in seinem einführenden Kapitel zu Hofmannsthals Komödien in HH, S. 227f. Bezeichnend für Hofmannsthals Interesse an der Commedia dell'Arte ist, dass er bereits 1893 das Fragment eines Versdialogs zwischen "Arlecchino und Don Juan" niederschreibt, das er möglicherweise als Puppenspiel konzipiert und in dem sich auch Bezüge zum Wiener Kasperletheater feststellen lassen. Vgl. SW XXI Dramen 19, S. 12, und den Kommentar von Mathias Mayer auf S. 183-185.

3 Roger Bauer, Hugo von Hofmannsthal und die venezianische Komödientradition. In: Literaturwissenschaftliches Jahrbuch 27, 1986, S. 105-122; s. auch Ders., "Der Impresario von Smyrna«. Ein Fragment aus Hofmannsthals Nachlaß. In: Das fremde Wort. Studien zur Interdependenz von Texten. Festschrift für Karl Maurer zum 60. Geburtstag. Hg. von Ilse Nolting-Hauff und Joachim Schulze. Amsterdam 1988, S. 376-393.

4 Eine Ausnahme ist Elena Raponis aufschlussreiche Monografie, in welcher die Autorin im Rahmen ihrer Auseinandersetzung mit Hofmannsthal und Italien auch Goldoni zwei Kapitel widmet: Dies., Hofmannsthal e l'Italia. Fonti italiane nell'opera poetica e teatrale di Hugo von Hofmannsthal. Mailand 2002, S. 89-99 und S. 219-288.

5 In Hofmannsthals Bibliothek sind neben einem teilweise unaufgeschnittenen Exemplar von Goldonis »Diener zweier Herren" auf Deutsch (Reclam 1873) und einem vermissten, bloß noch auf der Karteikarte aufgenommenen Exemplar von "Der Impresario von Smyrna" (Reclam 1881) vier Ausgaben von "Commedie scelte" von Goldoni im Original (Le Monnier, Firenze 1896 und 1897, Hoepli, Milano 1902 und Sonzogno, Milano 1902) verzeichnet. In den ersten beiden Ausgaben sind Anstreichungen und Anmerkungen von Hofmannsthals 
Beitrag auch die andere, ebenfalls mit Venedig verbundene Quelle von "Cristinas Heimreise" streifen, nämlich eine Episode aus dem zweiten Band von Casanovas Lebenserinnerungen. ${ }^{6}$ Für den vorliegenden Aufsatz erscheint dabei von besonderem Interesse, wie sich Hofmannsthals Casanova von dem venezianischen Verführer seiner literarischen Vorlage absetzt. Im Fokus des Beitrags steht daher die Frage, wie schöpferisch Hofmannsthal in "Cristinas Heimreise« in erster Linie mit Goldoni, aber auch mit seiner anderen Quelle umgegangen ist und ob er womöglich an dieser produktiven Rezeption des literarischen Venedig im 18. Jahrhundert 'gescheitert ist. Dabei wird das 'Scheitern` als eine sich auch ins Positive wendende Misslingenserfahrung verstanden, durch die sich neue künstlerische Möglichkeiten für Hofmannsthals Komödie eröffnen. ${ }^{7}$

\section{Hofmannsthals Goldoni}

Mit Goldoni kommt Hofmannsthal schon früh in Berührung: Nachdem er die Stücke des Venezianers seit den 1890er Jahren im Wiener Burgtheater gesehen hat, widmet er sich ab 1907 der Lektüre von Goldonis Komödien - einer Lektüre, die ihn über mehrere Jahre hindurch und bis 1925 begleiten wird. ${ }^{8}$ Und ebenfalls im Jahr 1907, in dem sich auch Goldonis Geburt zum 200. Mal jährt, liest Hofmannsthal Philippe Monniers Buch »Venise au XVIII siècle«, ein Geschenk von Harry Graf Kessler, in dem sowohl die Commedia dell'Arte als auch die Werke des venezianischen Komödiendichters ausführlich behandelt werden. ${ }^{9}$

Hand zu finden. Lesedaten lassen sich für die Jahre 1907, 1913, 1916 und schließlich 1922 festhalten. Vgl. SW XL Bibliothek, S. 251-253.

6 Hofmannsthal hatte die zwischen 1907 und 1909 erschienene Ausgabe gelesen: Giacomo Casanova, Erinnerungen. Übersetzt und eingeleitet von Heinrich Conrad. München/ Leipzig: Georg Müller. Vgl. SW XI Dramen 9, S. 419. Keiner der 13 Bände dieser Ausgabe hat sich in seiner Bibliothek erhalten. Neben einer Taschenbuchausgabe aus dem Jahr 1925 ist allerdings noch die französische Ausgabe von Casanovas Memoiren vorhanden (Paris 1880), vgl. SW XL Bibliothek, S. 126.

7 Vgl. dazu das Exposé der im vorliegenden Jahrbuch dokumentierten Tagung der Hugo von Hofmannsthal Gesellschaft, die den bezeichnenden Titel trug: "Hofmannsthals Komödie des Scheiterns" (http://hofmannsthal.de/hugo/wp-content/uploads/2016/12/HofmannsthalTagung_2017_Flyer.pdf [Zugriff: 31.10.2018]). Zum facettenreichen Begriff des 'Scheiterns vgl. auch das entsprechende Lemma von H. Rath in Historisches Wörterbuch der Philosophie, Basel 1971ff., Bd. 8, S. 1245f.

8 Vgl. dazu auch Raponi, Hofmannsthal e l'Italia (wie Anm. 4), S. 258.

9 Philippe Monnier, Venise au XVIII ${ }^{e}$ siècle. Paris 1907, vgl. besonders die Kapitel VIII und IX (»Le théâtre vénitien et la comédie italienne« und »La comédie de Goldoni«, S. 190- 
Monnier zufolge lebt die ‘Comédie italienner bei Goldoni weiter ${ }^{10}$ und eben diese Kontinuität wird in Hofmannsthals Goldonirezeption besonders relevant sein. Doch noch wesentlicher wird ihm die Grundidee sein, die Monniers Buch beseelt: Die Kernabsicht dieses Buches sei nämlich, wie gleich in der Einleitung beteuert wird, »d'étudier [une] nuance d'âme [qui fut heureuse] «11 - eine fröhliche Stimmungsnuance zu untersuchen, in der sich das Venedig des 18. Jahrhunderts für Monnier zugleich verkörpert und dematerialisiert und die auch Hofmannsthal in seinen Komödien atmosphärisch greifbar werden lassen möchte. ${ }^{12}$ Vor diesem Hintergrund wird Hofmannsthals Interesse für die venezianische Komödientradition in den ersten Jahren des 20. Jahrhunderts rege in dem Moment, als er sich in der für ihn neuen Gattung der Komödie in Prosa versucht, für die ihm nun u.a. Goldoni zum Modell wird. Sehr wahrscheinlich steht das auch mit Hofmannsthals in dieser Zeit sich anbahnender enger Zusammenarbeit mit Max Reinhardt in Zusammenhang. In diesen Jahren hatte Reinhardt die Commedia dell'Arte bzw. europäische Komödienautoren wie Goldoni, Gozzi und Molière für sich entdeckt und im deutschsprachigen Raum - und nicht zuletzt in Österreich - auf innovative Weise inszeniert. ${ }^{13}$ Nicht zufällig wird Carlo Gozzis »Turandot« in einer Neubearbeitung von Karl Vollmoeller bereits 1911 im Deutschen Theater Berlin von Reinhardt auf die Bühne gebracht. Darauf folgen 1912 "Ariadne auf Naxos" im Königlichen Hoftheater Stuttgart und 1924 Goldonis »Diener zweier Herren«. Dieses letzte Stück, mit dem Reinhardt sowohl das Theater in der Josefstadt in Wien als auch die Berliner Komödie eröffnet, wird er zwei Jahre später auch bei den von ihm zusammen mit Hofmannsthal gegründeten Salz-

258). Das Buch - mit zahlreichen Anstreichungen und Annotationen von Hofmannsthals Hand - ist noch in seiner Bibliothek vorhanden. Das Goldoni-Kapitel las Hofmannsthal im Juli 1907 und im August 1916 (vgl. SW XL Bibliothek, S. 480f.).

10 Vgl. etwa den vierten Absatz des zitierten Goldoni-Kapitels betitelt "La Commedia dell'arte dans la comédie de Goldoni«.

11 Ebd., S. 22.

12 Hofmannsthals Monnier-Lektüre beeinflusst außerdem den Schauplatz Venedig im "Andreas«-Fragment, wie den vorbereitenden Notizen zu diesem Roman zu entnehmen ist (vgl. SW XXX Roman, insbes. S. 7-22).

13 Wie der Ausstellungskatalog "Max Reinhardt und die Welt der Commedia dell'arte" (hg. von Edda Leisler und Gisela Prossnitz. Salzburg 1970) durch Texte und Bilder dokumentiert. 
burger Festspielen aufführen. ${ }^{14}$ Abgesehen von den Salzburger Festspielen ist Hofmannsthal an vielen Projekten beteiligt, in denen der Geist der Commedia dell'Arte im Sinne Reinhardts wiederbelebt werden soll: In Zusammenarbeit mit Reinhardt und Strauss entsteht die Oper »Ariadne auf Naxos", in deren Mittelpunkt die Gegenüberstellung von Opera seria und Commedia dell'Arte steht, und er schreibt auch einen szenischen Prolog zu Reinhardts Bearbeitung von Goldonis »Diener zweier Herren ${ }^{15}{ }^{15}$ Beiträge, die verschiedene Aspekte der Commedia dell'Arte thematisieren, erscheinen außerdem in diesen Jahren in den Hofmannsthal wohlbekannten »Blättern des deutschen Theaters « ${ }^{16}$ Hinzu kommt, dass die Commedia dell'Arte und Goldoni nicht erst durch Reinhardt in Wien ankommen, sondern spätestens seit dem 18. Jahrhundert fest zu der Wiener Theatertradition gehören, was in Hofmannsthals GoldoniRezeption sicher eine Rolle gespielt hat. Denn zu Hofmannsthals Zeiten sind die 'fremder europäische Tradition der Commedia dell'Arte und das reinheimischer Wiener Volksstück in Wien bereits so verflochten, dass sie nicht wirklich voneinander zu trennen sind. Schon zu Goldonis Lebzeiten wurden seine Komödien zuerst von Wien aus (etwa durch den in dieser Stadt geborenen und hier tätigen Schauspieler Antonio Sacchi) dem deutschsprachigen Raum bekannt gemacht. Die Stücke des venezianischen Komödiendichters wurden nicht nur in Wien häufig inszeniert, sondern es werden in der Hofbühne unter der Leitung des Grafen Giacomo Durazzo sogar Pläne gehegt, Goldoni als Hofdichter ans Burgtheater zu berufen, was dann aber nicht mehr zustande kam. ${ }^{17} \mathrm{Zu}$ betonen ist dabei, dass Goldoni im deutschsprachigen Raum von Anfang an und bis zu Hofmannsthals und Reinhardts Rezeption vorrangig nicht

14 Vgl. dazu die Studie von Norbert Christian Wolf, Eine Triumphpforte österreichischer Kunst. Hugo von Hofmannsthals Gründung der Salzburger Festspiele. Salzburg 2014.

15 Dieser Prolog ist in der Kritischen Ausgabe (SW XVII Dramen 15, S. 310-322) und auch im Ausstellungskatalog "Max Reinhardt und die Welt der Commedia dell'arte" (wie Anm. 13) abgedruckt (S. 6).

16 Besonders in dem Heft von 1911 sind fast ausschließlich Beiträge zu Gozzi und der Commedia dell'Arte zu finden, vgl. SW XXIV Operndichtungen 2, S. 66, FN 33.

17 Vgl. Karl Maria Grimme, Goldoni in Austria. In: Studi goldoniani. Hg. von Vittore Branca und Nicola Mangini. Venedig/Rom 1960 (Atti del convegno internazionale di studi goldoniani, Venezia 28 settembre - 1 ottobre 1957), Bd. 2, S. 291-296, hier S. 293. Vgl. außerdem Camillo Valerian Susan, Carlo Goldoni. In: Separatabdruck aus der Österreichischen Rundschau. Bd. X, Heft 4, 15. Februar 1907. Hg. von Alfred Freiherrn von Berger und Karl Glossy, S. 288-294, insbes. S. 292ff., auf denen der Autor dokumentiert, wie oft Goldonis Komödien von 1751 bis zu Hofmannsthals Zeiten in Wien inszeniert wurden. 
als der raufklärerischer Theaterreformer wahrgenommen wurde, der das Stegreiftheater zugunsten einer festen Bühne abgesetzt und neu gedacht hat, sondern als der Dichter der Commedia dell'Arte (auch `Commedia all'italianar genannt), der er zum Teil ja durchaus war. Auch allgemein wurde Goldoni außerhalb Italiens in erster Linie als Vertreter eines "mimischen und rhythmischen Theaters" aufgefasst, während die eher aufklärerischen, moralisch-pädagogischen Aspekte seines Komödienwerks in den Hintergrund traten. ${ }^{18}$ Demzufolge wurde Goldoni von Anfang an sowohl in den Augen der Schauspieler wie des Publikums nicht etwa als der Begründer des modernen komischen Theaters, sondern als der Fortsetzer des alten Maskenspiels betrachtet und entsprechend sehr frei interpretiert. Auch aus diesem Grund kann man annehmen, dass der frühe und starke Erfolg von Goldonis Komödien in Wien ${ }^{19}$ nicht zuletzt auf deren Verwandtschaft mit dem Wiener Volksstück zurückgeht - eine Verwandtschaft, die nicht zuletzt im Improvisationstheater ihre Wurzeln hat. Während die Rezeption Goldonis im Europa des 18. Jahrhunderts gut erforscht ist, ist die der beiden folgenden Jahrhunderte von der Forschung eher vernachlässigt worden, ${ }^{20}$ was sich auch in Forschungs-

18 Das schließt natürlich nicht aus, dass Goldoni im deutschsprachigen Raum gerade in dem Moment, in dem die Gründung eines Nationaltheaters aktuell wurde, als Modell für ein neues komisches Theater wahrgenommen wurde. Vor diesem Hintergrund wurde die erste Übersetzung von Goldonis Werken ins Deutsche (44 Komödien) durch Justus Heinrich Saal gerade von Lessing angeregt. Nicht zufällig standen auf dem Spielplan von Lessings Hamburger Nationaltheater bereits 1760 mehrere Komödien von Goldoni. Vgl. Nicola Mangini, La fortuna del teatro goldoniano in Europa nel Settecento. Quadro di riferimento dei principali percorsi. In: Carlo Goldoni 1793-1993. Atti del Convegno del Bicentenario. Hg. von Carmelo Alberti und Gilberto Pizzamiglio. Venedig 1995, S. 89-97, hier S. 95. Vgl. dazu auch den Beitrag von Nicola Mangini, La fortuna di Goldoni nel mondo. In: Unità e diffusione della civiltà veneta. Relazioni e comunicazioni del convegno degli scrittori veneti, Gorizia ottobre 1974. Hg. von Ugo Fasolo und Nereo Vianello, Associazione degli scrittori veneti, s.l. 1975, S. 167-173, hier S. 169.

19 In Wien und in deutscher Sprache erscheint zum Beispiel im Jahr 1751 die erste Druckversion einer Goldoni-Komödie in Europa. Es handelt sich um "La vedova scaltra" (»Die schlaue Wittib«. Eine, von dem berühmten Advocaten zu Venedig Sigr. Carlo Goldoni verfertigte galante Comödie aus dem Italiänischen desselben auf das Wienerische Theater übersetzt und eingerichtet von J.A.D.S, gedruckt bey Joh. Peter Gehlen, Kaiser. Königl. Hofbuchdrucker, Wien 1751).

20 Wie auch Rita Unfer Lukoschik in ihrem Beitrag berichtet: Aspetti della ricezione di Goldoni in Germania (1743-2008). In: Ein europäischer Komödienautor. Carlo Goldoni zum 300. Geburtstag/Carlo Goldoni commediografo europeo nel terzo centenario della nascita. Hg. von Richard Schwaderer, Rita Unfer Lukoschik und Friedrich Wolfzettel. München 2008, S. 97-117, hier S. 97f. 
lücken bezüglich der Goldonirezeption in der Wiener Moderne und bei Hofmannsthal niederschlägt.

Eine für die Goldoni-Studien am Anfang des 20. Jahrhunderts in Wien zentrale Figur war sicher der aus Zadar stammende, wenig bekannte Italienischlektor Edgardo Maddalena, der ein Schüler und Mitarbeiter vom ebenfalls in Dalmatien gebürtigen Romanistikprofessor Adolf Mussafia war, bei dem Hofmannsthal während seines Romanistikstudiums mehrere Veranstaltungen besucht hatte. ${ }^{21}$ Auch wenn nicht dokumentiert ist, dass Hofmannsthal in Maddalenas Seminaren gesessen hat, kann man davon ausgehen, dass er den Lektor kannte. Denn dieser hatte die Grammatik der italienischen Sprache von Adolf Mussafia, die ein Standardwerk war und die auch Hofmannsthal selbst benutzt hatte, ergänzt und mit einer dann in mehreren Auflagen erschienenen Textanthologie versehen. ${ }^{22}$ Maddalena stammte aus einer theaterbegeisterten dalmatischen Familie ${ }^{23}$ und hatte, von Mussafia betreut, mit einer Magisterarbeit zu Giuseppe Barettis Goldonikritik sein Studium an der Universität Wien abgeschlossen. ${ }^{24}$ Der Italianist und spätere Germanist Maddalena entwickelte sich zu einem ausgewiesenen Goldoni-Forscher, der für spätere Goldoni-Studien ein unverzichtbarer Bezugspunkt wurde. ${ }^{25}$ Gerade in Hofmannsthals Studienjahren hatte ihm Mussafia nicht nur den italienischen Sprachunterricht anvertraut, sondern auch Litera-

21 Wie aus Hofmannsthals Studienbuch hervorgeht, hatte der Dichter im Wintersemester 1897/1898 und im Sommersemester 1898 u.a dessen Seminare zur italienischen Literatur und Grammatik sowie ein Seminar zu Molière besucht. Für diese Information danke ich Frau Dr. Olivia Varwig.

22 "Raccolta di prose e poesie italiane annotate ad uso dei tedeschi« (Wien 1894). Außerdem werden drei von Maddalena herausgegebene Goldoni-Bände auch in der Bibliografie von dem für Hofmannsthal zentralen Venedigbuch Monniers zitiert (Una diavoleria di titoli e cifre, Neapel 1900; Goldonis Lettere inedite. Neapel 1901; Moratin e Goldoni. Capodistria 1904, vgl. Monnier, Venise au XVIII siècle [wie Anm. 9], S. 394 und S. 396).

23 Wie der Italianist Attilio Gentile berichtet, war Maddalenas Vater Giacomo vor 1850 ein berühmter Harlekin im Karneval gewesen, während zwei seiner Brüder Schauspieler wurden. Edgardo kannte Goldonis Werke seit seiner Kindheit und pflegte in seiner Familie oft Theater zu spielen. Vgl. Attilio Gentile, La fortuna di Carlo Goldoni fuori d'Italia nelle ricerche di Edgardo Maddalena. In: Atti del Reale Istituto Veneto di Scienze, Lettere ed Arti, Anno Accademico 1939-1940, Bd. XCIX, Parte II, S. 357-375, hier S. 358f.

24 Edgardo Maddalena, Baretti's Urtheil über Carlo Goldoni (1890). Das ungedruckte Manuskript befindet sich in der Bibliothek des Goldoni-Hauses in Venedig (Biblioteca di Studi Teatrali). Geplant ist ein an der Universität Venedig angesiedeltes Forschungsprojekt zu Maddalena, in dessen Rahmen auch ungedruckte Quellen wissenschaftlich bearbeitet und zugänglich gemacht werden sollen.

25 Vgl. dazu Gentile, La fortuna di Carlo Goldoni fuori d'Italia nelle ricerche di Edgardo Maddalena (wie Anm. 23), passim. 
turseminare, u.a. zu Goldoni und Gozzi. ${ }^{26}$ Es mag kein Zufall gewesen sein, dass Hofmannsthal schon 1898 - in seiner Studienzeit also - unter seinen Lektüren Goldoni erwähnt. ${ }^{27}$ Diese Lektüren wird er im Spannungsverhältnis von Verehrung und Distanz einige Jahre später auch mit Bezug auf sein eigenes Komödienwerk wiederaufnehmen.

\section{Für und gegen Goldoni „Mirandolina« und "Cristinas Heimreise«}

Ab 1907 intensiviert Hofmannsthal seine Goldoni-Lektüren, denn das Interesse an den Komödien des venezianischen Theaterreformers tritt bei ihm in dem Moment in den Vordergrund, als er sich auch selbst der Gattung Komödie widmet. Genau in dieser Zeit entsteht die erste vieraktige Fassung von "Cristinas Heimreise«, die noch den Titel »Florindos Werk « trägt und ein Stück für das Sprechtheater ist. Sowohl in dieser ersten als auch in den späteren, unter dem Titel "Cristinas Heimreise« umgearbeiteten Fassungen lassen sich Bezüge zu Goldoni feststellen. ${ }^{28}$ Besonders relevant erscheinen dabei die Verbindungen zu Goldonis Komödie »La locandiera«, die Hofmannsthal in einer Originalausgabe der "Commedie scelte« von 1902 liest $^{29}$ - ein Exemplar, das er, wie bereits erwähnt, besitzt und mit Anmerkungen versieht, die sich auf »Cristinas Heimreise" beziehen. Hofmannsthals Annotationen stellen Parallelen zwischen der Haltung von Goldonis Mirandolina und der seiner Cristina her, konzentrieren sich dann auf die Figur des Ritters von Ripafratta und heben schließlich das immerfort sich ergebende $»$ spiessige[ ] « Gespräch ${ }^{30}$ zwischen dem Ritter, dem Grafen und dem Marquis hervor.

Die erste Stelle, die Hofmannsthal in seiner Ausgabe unterstreicht, befindet sich in der neunten Szene des ersten Aktes, in der Mirandolina

26 Vgl. dazu auch Raponi, Hofmannsthal e l'Italia (wie Anm. 4), S. 55 f.

27 Ebd.

28 Bezeichnend für diese Bezüge ist, dass die Hauptfigur der noch titellosen Komödie infolge von Hofmannsthals Lektüre von Goldonis Komödie "Gli amori di Zelinda e Lindoro» im April 1908 nicht Florindo hieß, sondern Lindor, vgl. SW XI Dramen 9, S. 419.

29 Carlo Goldoni, Commedie scelte. Annotate da Adolfo Padovan, con un proemio di Giuseppe Giacosa su "L'arte di Carlo Goldoni«. Mailand 1902. Ein Buchexemplar ist in Hofmannsthals Bibliothek vorhanden, vgl. SW XL Bibliothek, S. $253 f$.

30 Ebd., S. 253. 
in einem Monolog ihre herausfordernde Absicht kundgibt, den widerspenstigen Ritter erobern zu wollen. Jenseits aller wahren oder fingierten Aufrichtigkeit, die sie doch in der ganzen Komödie beteuert, enthüllt sie hier auch ihre kokette Natur:

Mirandolina allein. [...] Aber er wird sie [eine Frau, die es ihm zeigt] finden. Und ob er sie finden wird! Wer weiß, ob er sie nicht schon gefunden hat? Gerade mit solchen lege ich mich gerne an. Die, die mir nachlaufen, sind mir sehr bald lästig. Der Adelsstand ist nichts für mich. Den Reichtum schätze ich und auch wieder nicht. Mein ganzes Vergnügen besteht darin, mich verwöhnt, umworben und angehimmelt zu sehen. Dies ist meine Schwäche, wie es die Schwäche fast aller Frauen ist. Ans Heiraten denke ich nicht einmal im Traum. Ich brauche niemanden. Ich lebe anständig und genieße meine Freiheit. Ich komme mit allen gut aus, aber ich verliebe mich niemals in einen. ${ }^{31}$

An dieser Stelle wird klar, dass Mirandolina noch - bei allen Unterschieden vor allem in der psychologischen Charakterisierung dieser Figur bei Goldoni - auf der Folie der koketten Magd Colombina entworfen ist. Tatsächlich lassen sich hinter den Figuren Goldonis noch immer die Masken der Commedia dell'Arte wiedererkennen, denn in seinem Komödienwerk geht es nicht etwa um eine Abschaffung der Commedia dell'Arte, sondern um eine Theaterreform, die sowohl auf eine schriftliche Fixierung des Komödientextes (im Unterschied zum Stegreifspiel) als auch auf eine Entwicklung und Analytik der Charaktere (im Gegensatz zu den Masken) hinausläuft. ${ }^{32}$ In diesem Sinne erinnern in Goldonis "Locandiera" neben Mirandolina sowohl der Diener Fabrizio als auch der Ritter von Ripafratta an typische Masken des Improvisationsthea-

31 Carlo Goldoni, Mirandolina, Übersetzung und Nachwort von Annika Makosch. Stuttgart 1991, S. 18. Die Stelle, die Hofmannsthal in der Originalausgabe unterstreicht, lautet: "Ma la troverà. La troverà. $\mathrm{E}$ chi sa che non l'abbia trovata? Con questi per l'appunto mi ci metto di picca. Quei che mi corrono dietro, presto presto mi annoiano. La nobiltà non fa per me. La ricchezza la stimo e non la stimo. Tutto il mio piacere consiste in vedermi servita, vagheggiata, adorata. Questa è la mia debolezza, e questa è la debolezza di quasi tutte le donne. A maritarmi non ci penso nemmeno; non ho bisogno di nessuno; vivo onestamente, e godo la mia libertà. Tratto con tutti, ma non m'innamoro mai di nessuno« (SW XL Bibliothek, S. 253).

$32 \mathrm{Zu}$ Goldonis reformatorischem Konzept vgl. das Nachwort von Annika Makosch zu der neuesten deutschen Fassung von Goldonis »Locandiera" in Mirandolina (wie Anm. 31), S. $107-116$. 
ters - einerseits an Brighella und Harlekin, anderseits an den Kapitän. Bei Goldoni stehen allerdings nicht mehr der Sprachwitz und das Körperspiel im Vordergrund, vielmehr gehen diese spielerischen Elemente mit der genuin aufklärerischen Idee Hand in Hand, dass man auch durch das Lachen die Sitten verbessern kann. In Goldonis Vorwort zu "Mirandolina« (»L'autore a chi legge») wird nicht zufällig gleich beim ersten Satz die Moral des Stückes erwähnt: Unter seinen Komödien sei "La locandiera" - so der Autor an den Leser - »die moralischste, die nützlichste und die lehrreichste «. ${ }^{33}$ Bezeichnenderweise endet das Stück ebenfalls mit Worten von Mirandolina, die den `Nutzen hervorheben - wobei der Leser bis zum Schluss natürlich nicht sicher sein kann, ob die Rede von der Moral nicht auch bloß Teil des Theaterspiels ist:

Mirandolina Diese Zusicherungen werden mir im Rahmen der Schicklichkeit und des Anstands teuer sein. Mit dem Stand möchte ich auch meinen Lebenswandel ändern. Die Herrschaften aber mögen aus dem, was sie gesehen, zu ihrem Vorteil und zur Sicherheit ihrer Herzen Nutzen ziehen. Sollten Sie je Veranlassung haben, zu zweifeln, nachzugeben, sich zu ergeben, erinnern Sie sich und gedenken Sie der lehrreichen Ränke der Gastwirtin Mirandolina. ${ }^{34}$

Dass diese Figur sonst von bürgerlich-moralischen Werten nicht viel zu halten scheint, lässt umso mehr an der von Mirandolina im Stück immer wieder beteuerten >Aufrichtigkeit‘ zweifeln. Man braucht sich nur ihre bereits zitierten Worte ins Gedächtnis zu rufen: »Mein ganzes Vergnügen besteht darin, mich verwöhnt, umworben und angehimmelt zu sehen. [...]. Ans Heiraten denke ich nicht einmal im Traum ${ }^{35}$ Der Punkt ist, dass sich Mirandolina genau in dieser spielerisch-provokativen Haltung, die im Geiste der Commedia dell'Arte die bürgerliche Moral des Stückes relativiert, von Hofmannsthals Cristina unterscheidet. Im dritten Akt der ersten Fassung, auf den Hofmannsthal in seinen Annotationen mit Bezug auf eben diese Stelle verweist, vernimmt man aus Cristinas Mund ganz andere Worte über die Ehe:

33 Vgl. Carlo Goldoni, La locandiera. Hg. von Sara Mamone und Teresa Megale. Venedig 2007, S. 123 (Übersetzung der Verfasserin).

34 Goldoni, Mirandolina (wie Anm. 31), S. 105.

35 Ebd., S. 18. 
Gut ist die Ehe. In ihr ist alles geheiligt. Das ist kein leeres Wort. Das ist die Wahrheit. Es führen's viele im Munde, aber wer's einmal begriffen hat, der versteht's. (SW XI Dramen 9, S. 248)

Wie aus diesen Worten zu erkennen, ist Hofmannsthals Cristina strenggenommen von viel mehr `Morak und `Aufrichtigkeit gekennzeichnet, als das bei Goldonis Mirandolina der Fall ist. Auch jenseits der Figur von Cristina wird die Relevanz der Moral durch die vorrangige Rolle der Ehe in Hofmannsthals Komödie bestätigt. Doch ist dabei nicht die Ehe als zentraler Wert einer bürgerlichen Moral oder gar als klassisches Komödienthema gemeint: Vielmehr wird die Ehe hier als Sakrament, ja als "Mysterium« aufgefasst, was paradoxerweise gerade von der Figur des Florindo behauptet wird, einer Figur, die eigentlich als Verführer am wenigsten an moralische Werte gebunden sein sollte:

FloRINDo [...] Zeig mir einen Mann und eine Frau, die einander wert sind: wie sie zusammengekommen sind, danach will ich nicht fragen. Aber daß sie beieinander zu verbleiben vermögen, das ist wundervoll. Das geht über die gemeinen Kräfte. Das ist ein Mysterium - kaum zu fassen ist es. - Und darum bitte ich mir Respekt aus davor, so wie ich ihn selber im Leibe habe. (SW XI Dramen 9, S. 357) ${ }^{36}$

Mit dieser religiösen Auffassung der Ehe, die aus dem Wort "Mysterium« hervorgeht, nimmt das Stück von bürgerlich-konservativen Werten Abstand, um sich aus dem Alltag heraus in eine höhere Dimension zu begeben. Und diese Distanz vom Bürgerlichen ist auf einer anderen Ebene auch der Grund, aus dem Geldfragen, die bei Goldoni von Anfang an ständig thematisiert werden, ${ }^{37}$ aus Hofmannsthals Komödie völlig verschwinden. Diese sprosaische Seite von Goldonis »Locandiera«

36 Dieses Zitat bezieht sich auf die zweite Fassung der Komödie, wobei die moralische Seite von Hofmannsthals Verführer sowie dessen Einsatz für die Ehe schon in der ersten Fassung eindeutig sind. Nicht zufällig zeigt sich Florindo schon hier von der Geschichte von Philemon und Baucis, dem treuen Ehepaar aus Ovids "Metamorphosen«, sehr angetan, vgl. SW XI Dramen 9, S. 258 .

37 Man denke nur an die allererste Szene der Komödie, in der sich der Marquis von Forlipopoli und der Graf von Albafiorita eigentlich nur über den gekauften Titel und das Vermögen des Grafen unterhalten sowie über die Tatsache, dass der Marquis im Gegensatz zum Grafen zwar einen geerbten Titel besitze, aber alle Geldsubstanzen verloren habe. 
kann Hofmannsthal nicht entgangen sein, wenn er in seiner oben erwähnten Annotation gerade das immerfort sich ergebende sspießige $\mathrm{Ge}$ spräch zwischen dem Ritter, dem Grafen und dem Marquis in Goldonis Stück unterstreicht. Wenn Hofmannsthal in seinen Komödien dagegen beabsichtigt, »das Einsame u. das Sociale, das Mystische u. das Dialecte, Sprache nach innen u. Sprache nach außen « zusammenzufassen, ${ }^{38}$ dann steht auch in "Cristinas Heimreise " nicht mehr das Bürgerliche, sondern ein sSoziales` im Mittelpunkt, das ein mystisches Element in sich trägt. Wenn man diesen einmal mehr produktiven Umgang mit dem bürgerlichen Goldoni im Auge behält, kann man - bei aller Verehrung gegenüber dem venezianischen Komödiendichter - auch Hofmannsthals Goldoni-Kritik nachvollziehen. Wie der Dichter 1921 in seinem »Buch der Freunde« schreibt, habe Goldoni zwar ein dichterisches Talent, doch im Wesentlichen eine Philisternatur: "Goldoni: dichterische Hand, aber Eingeweide eines Philisters«, notiert er apodiktisch. ${ }^{39}$ Man kann leicht erraten, dass damit die bürgerliche, alltägliche Seite von Goldonis Werk angeprangert werden soll. Wenn die Komödie, wie Hofmannsthal im "Buch der Freunde" ausdrücklich schreibt, nicht "schmackhaft sein [kann] ohne einen Hauch von Mystizismus « ${ }^{40}$, dann ist es gerade dieser Mystizismus, auf den Hofmannsthal im Gegensatz zu Goldoni in seiner Komödie setzt. ${ }^{41}$ Über die shöhere Aufgabe seiner Komödie "Cristinas Heimreise « äußert sich Hofmannsthal deutlicher in einem Interview vom 23. April 1926 im »Neuen Wiener Journal«:

Das ewige Zueinanderfinden, das Sichtrennen und Wiederzusammenkommen ist die Idee von >Christinas [!] Heimreiser. Für Florindo ist die Begegnung mit Christina nur eine Zufälligkeit; der Kapitän, der das Mädchen schließlich heiratet, empfindet diese Begegnung als etwas Höheres. Er hebt sie aus dem Alltag heraus ins Größere, Bleibende. Und hier liegt der tiefere Sinn, wenn Sie wollen: die Moral des Lustspiels. (SW XI Dramen 9, S. 834)

Gerade unter dem Vorzeichen dieser stieferen Moral hat auch der Kapitän, der mit seiner Zurückhaltung, Langsamkeit und Ungeselligkeit viele

38 Wie er am 22. August 1917 an Rudolf Pannwitz schreiben wird (vgl. BW Pannwitz, S. 34).

39 SW XXXVII Aphoristisches - Autobiographisches - Frühe Romanpläne, S. 54.

40 Ebd., S. 59.

$41 \mathrm{Im}$ "Buch der Freunde" wird die mystische Seite der Ehe, die "Cristinas Heimreise" beseelt, von Hofmannsthal mehrmals beteuert. Vgl. etwa ebd., S. 24f. (158 und 169). 
Gemeinsamkeiten mit Goldonis Ritter von Ripafratta aufweist ${ }^{42}$ und in dem auch der `Capitano der Commedia dell'Arte bereits im Namen eindeutig weiterlebt, ${ }^{43}$ doch eine völlig andere Einstellung zur Ehe als der frauenfeindliche Ritter. In einem Gespräch zwischen dem Kapitän und Florindo im dritten Akt der ersten Fassung nimmt Hofmannsthal die vierte Szene des ersten Akts von Goldonis "Locandiera" wieder auf und variiert seine Vorlage gerade in Hinsicht auf die Ehe. Bei Goldoni macht der Ritter - eine für Hofmannsthal sicher zentrale Gestalt, der seine dritte Annotation im Text gilt - dem Grafen und dem Marquis, die ihm die Ehe nahebringen möchten, anschaulich klar, dass er sich nie verliebt hat bzw. niemals verlieben wird. Seine Aussage bekräftigt der Ritter durch die Behauptung, dass er, der fatalerweise keine Nachkommenschaft hat, nicht einmal aus diesem, im Sinne der bürgerlichen Atmosphäre des Stückes doch triftigen Grund bereit ist, eine Beziehung mit einer Frau in Betracht zu ziehen:

RitTer Ich erwog es bereits mehrfach. Aber wenn ich bedenke, daß ich, um Kinder zu haben, eine Frau ertragen müßte, vergeht mir sofort die Lust. ${ }^{44}$

Ganz anders sieht die Reaktion von Hofmannsthals Kapitän in einem Gespräch mit Florindo im zweiten Akt aus. Auf Florindos Veranlassung »Sie müssen heiraten, Kapitän. Sie müssen Kinder haben « ${ }^{45}$ antwortet der redliche Kapitän mit folgenden, dem Geist von Goldonis Ritter diametral entgegengesetzten Worten: "Das bin ich willens, Herr, so hab' ich Ihnen gestern gesagt. Sie sehen mich sozusagen auf dem Wege dazu, Herr « ${ }^{46}$ Hofmannsthals Absicht ist es hier, wie er auch in einem Brief vom 29. Januar 1910 an Harry Graf Kessler schreibt, in den Figuren des Verführers und des Kapitäns einmal mehr im Einklang mit der `Morak des Lustspiels "naturhafte Polygamie und geheiligte Ehe als große Potenzen nebeneinander [und] in der Person ihrer Vertreter in ein freundschaftliches Verhältnis zu einander ${ }^{47}$ zu stellen. Auch wenn diese beiden

42 Beide Gestalten sind - um ein Goldoni-Wort zu benutzen - durch eine gewisse "rustichezza" (Ungeschliffenheit) charakterisiert.

43 Zur Figur des Capitano vgl. ausführlich Fausto de Michele, Guerrieri ridicoli e guerre vere; nel teatro comico del '500 e del '600 (Italia, Spagna e paesi di lingua tedesca). Florenz 1998.

44 Goldoni, Mirandolina (wie Anm. 31), S. 12.

45 SW XI Dramen 9, S. 226.

46 Ebd.

47 Ebd., S. 797. 
Potenzen laut Hofmannsthal »de pair« sein sollten, ${ }^{48}$ scheint die 'geheiligter Ehe in den Worten und Positionen der verschiedenen Figuren des Stückes am Ende doch die Oberhand zu gewinnen. Selbst die Figur des sorgenlosen und lebendigen Pedro entzieht sich nicht dem allgemeinen Lob der Ehe, und sie zeigt sich auf seine »drollig naive «9 Art sehr dankbar, heiraten zu können:

PEDRO Ich bin sehr glücklich, mich abermals die Hände zu schütteln mit einen Herrn, der so gut versteht die Heirat in europäischer Weise. Ich bin im Begriffe in den Ehestand hineinzutreten mit Hilfe der betreffenden Witwenfrau, und wir werden immer gedenken auf unseren Anstifter mit zudringlicher Dankbarkeit. (SW XI Dramen 9, S. 259)

In dieser Figur spiegelt sich in mehreren Hinsichten der typische Diener der Commedia dell'Arte wider: Wenn Pedros komische Sprache z.B. an die verschiedenen dialektalen Varianten des Stegreifspiels erinnert, ${ }^{50}$ so ruft auch die ausgeprägte Gestik dieser Figur das Körperspiel des alten Maskentheaters auf:

[Bühnenanweisung] Pedro horcht zu, amüsiert sich. Tanzt einen Tanz, der die Vision des unkultivierten Wilden hervorruft. [...] Pedro hält inne, nimmt eine sehr unbefangene Miene an, als hätte er keinen Augenblick die Haltung eines wohlerzogenen Europäers verlassen, lehnt an der Haustüre.

(SW XI Dramen 9, S. 205)

Doch lässt Pedro in "Cristinas Heimreise" nicht nur den alten `Diener zu Worte kommen, er personifiziert zugleich auch eine völlig neue Figur. Dies macht sich besonders in Pedros kulturkritischer Haltung gegenüber den europäischen Sitten bemerkbar, einer Haltung, die sich besonders in seinem »Verkennen der europäischen Lebenskomplikationen « ${ }^{51}$ äußert. Denn als Malaie ist er ein Fremdländer, ${ }^{52}$ der nicht nur diese Sitten

48 Ebd.

49 So bezeichnet Hofmannsthal Pedros Ausdrucksweise in einem Brief vom 8. Februar 1910 an Max Reinhardt, vgl. ebd., S. 800.

50 Annika Makosch erinnert z.B. daran, dass in der ersten Aufführung von Goldonis »Locandiera« der Diener Fabrizio noch Dialekt sprach (wie Anm. 31), S. 114.

51 So Hofmannsthal wörtlich im zitierten Brief an Reinhardt. Die gerade aus diesem »Verkennen" hervorgehende Komik erklärt der Autor als die dichterische Grundabsicht dieser Figur, vgl. SW XI Dramen 9, S. 800.

52 Auch wenn er, wie es im Text heißt, einen europäischen Vater hat und getauft ist, vgl. ebd., S. 285 und S. 313. Er selbst beschreibt sich als ein »junger ausländischer Europäer» 
als unaufrichtig entlarvt ${ }^{53}$ und für »künstlich" erklärt, ${ }^{54}$ sondern auch die Lebendigkeit verkörpert, die den sdekadenten Europäern abhandengekommen sei. In einem Brief an Harry Graf Kessler unterstreicht Hofmannsthal selbst die Neuheit dieser Figur, die in seinen Augen nicht zufällig auch »[m]anches vom Tiefsten des Stückes [aus]spricht.$_{.5}^{55}$ Somit ist Pedro - auch hier gegen den Geist von Goldonis bürgerlichen Komödien - ebenfalls eine Figur, in der sich eine stiefere Morak, ja ein »Hauch von Mystizismus ${ }^{56}$ verbirgt, eine Figur, in der sich, um Hofmannsthals eigene Worte zu benutzen, »das Geistige mit dem Weltstoff« vermählt. ${ }^{57}$

\section{Ein Fazit \\ Das Scheitern an Casanova oder die »erzkonservative Tendenz des Stückes"}

Auch der Verführer Florindo, der die Figur des Casanova wiederaufnimmt, entzieht sich in "Cristinas Heimreise", wie angedeutet, nicht der shöheren Moral des Stückes. Bekanntlich interessierte sich Hofmannsthal wie viele Autoren der Wiener Moderne für den venezianischen Frauenhelden aus dem 18. Jahrhundert und plante auch einen Casanova-Aufsatz. ${ }^{58}$ Eine Episode aus dem zweiten Band von Casanovas "Mémoires«, die wie bereits erwähnt 1907 bis 1909 von Heinrich Conrad ins Deutsche übertragen wurde und die Hofmannsthal, wie aus Briefdokumenten und wortgetreuen Exzerpten hervorgeht,${ }^{59}$ in eben dieser Über-

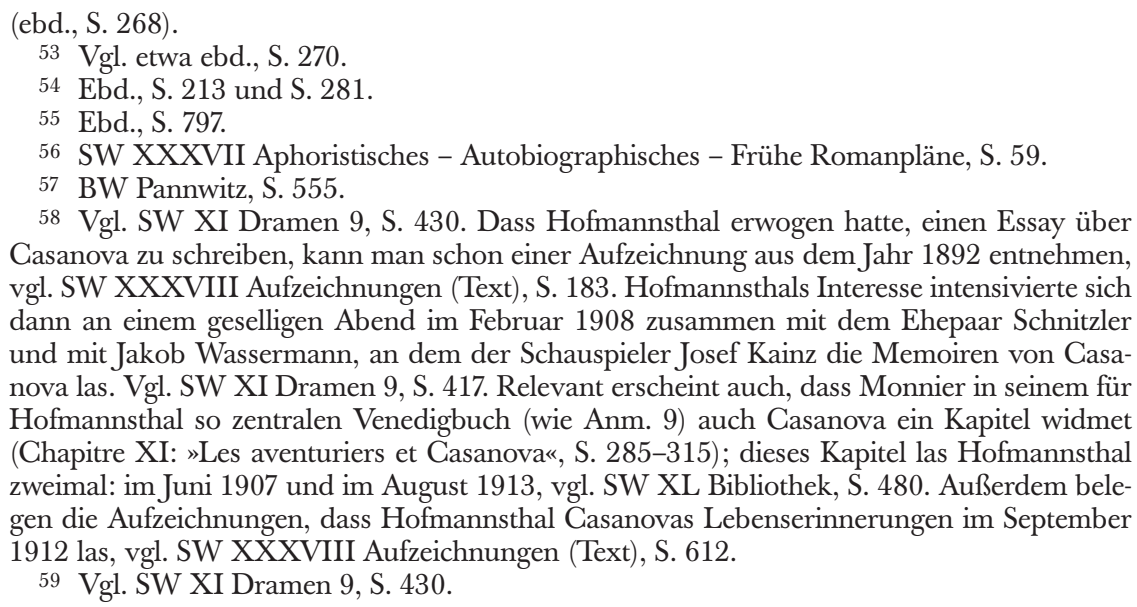

58 Vgl. SW XI Dramen 9, S. 430. Dass Hofmannsthal erwogen hatte, einen Essay über Casanova zu schreiben, kann man schon einer Aufzeichnung aus dem Jahr 1892 entnehmen, vgl. SW XXXVIII Aufzeichnungen (Text), S. 183. Hofmannsthals Interesse intensivierte sich dann an einem geselligen Abend im Februar 1908 zusammen mit dem Ehepaar Schnitzler und mit Jakob Wassermann, an dem der Schauspieler Josef Kainz die Memoiren von Casanova las. Vgl. SW XI Dramen 9, S. 417. Relevant erscheint auch, dass Monnier in seinem für Hofmannsthal so zentralen Venedigbuch (wie Anm. 9) auch Casanova ein Kapitel widmet (Chapitre XI: "Les aventuriers et Casanova«, S. 285-315); dieses Kapitel las Hofmannsthal zweimal: im Juni 1907 und im August 1913, vgl. SW XL Bibliothek, S. 480. Außerdem belegen die Aufzeichnungen, dass Hofmannsthal Casanovas Lebenserinnerungen im September 1912 las, vgl. SW XXXVIII Aufzeichnungen (Text), S. 612.

59 Vgl. SW XI Dramen 9, S. 430. 
setzung gelesen hatte, ist auch eine Quelle für "Cristinas Heimreise ${ }^{60}{ }^{60}$ Dass Hofmannsthal Casanovas >literarische Figur aus den "Mémoires" wiederaufnehmen, dass er aber auch eine neue Figur daraus machen möchte, geht besonders aus dem Dialog mit Harry Graf Kessler hervor, der die Entstehung von Hofmannsthals Komödie begleitet. Wenn Florindo für Hofmannsthal einerseits eine lebhafte Gestalt sein soll, die »blos [sic!] durch [ihre] Lebensfülle, durch [ihr] Temperament, die Dinge weiterbringe « ${ }^{61}$ so soll er andererseits »keineswegs identisch mit Casanova ${ }^{62}$ sein; in diesem Sinne vielmehr zugleich - wie Kesslers Tagebuch zu entnehmen ist - eine »Gegenfigur zu Figaro «. ${ }^{63}$ Tatsächlich stellt Florindo in den Absichten seines Autors auch eine durch und durch moralische Figur dar, die nicht mehr nur "Sklave des Gefühls « ${ }^{64}$ ist und sich wie alle anderen Figuren auch für die 'Heiligkeit` der Ehe einsetzt. So erweist sich Hofmannsthals Verführer als eine ambivalente Figur, die durch Lebensfülle, aber zugleich durch eine ausgeprägt konservative Haltung charakterisiert ist. Die - so der Autor wörtlich - »erzkonservative Tendenz des Stückes", die sich auch in Florindo bekundet, hat Hofmannsthal selbst in einem Brief an Max Reinhardt betont. ${ }^{65}$ Selbstverständlich ist hier ein 'Konservativismus` gemeint, der von bürgerlichen Werten Abstand nehmen möchte. Es möge am Ende dahingestellt bleiben, ob das konservative Denken, das im Stück greifbar wird, womöglich bereits spätere Entwicklungen von Hofmannsthals Werk und Denken vorwegnimmt - Entwicklungen, die 1927 in Hofmannsthals Schrifttumsrede vielleicht ihren prägnantesten theoretischen Ausdruck finden werden. Sicher ist allerdings, auch im Hinblick auf die von Hofmannsthal beteuerte »erzkonservative Tendenz des Stückes", dass er in seiner Komödie an der Rezeption von Goldoni und Casanova - durchaus schöpferisch 'gescheitert ist.

60 Die Quelle ist in SW XI Dramen 9, S. 430-453 abgedruckt, auch die folgenden Zitate in SW XI.

61 So liest man in Kesslers Tagebuch (Eintrag vom 13. März 1908), vgl. ebd., S. 733.

62 Hofmannsthal am 29. Januar 1910 an Kessler, vgl. ebd., S. 796.

63 Dabei ist selbstverständlich Beaumarchais' Figaro gemeint.

64 So bezeichnet sich Casanova in seinen Memoiren, vgl. ebd., S. 442.

65 Ebd., S. 801.

106 Cristina Fossaluzza 